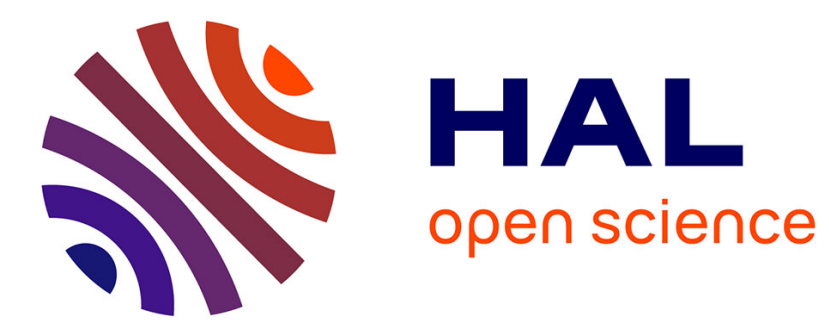

\title{
Enhanced Spectrally Aware RF front end Receiver under Non-linearity
}

Nicolas Grollier, Sébastien Houcke, Michaël Pelissier

\section{To cite this version:}

Nicolas Grollier, Sébastien Houcke, Michaël Pelissier. Enhanced Spectrally Aware RF front end Receiver under Non-linearity. ICC 2018: IEEE International Conference on Communications, May 2018, Kansas City, United States. 10.1109/ICC.2018.8422207 . hal-01735642

\section{HAL Id: hal-01735642 \\ https://hal.science/hal-01735642}

Submitted on 16 Mar 2018

HAL is a multi-disciplinary open access archive for the deposit and dissemination of scientific research documents, whether they are published or not. The documents may come from teaching and research institutions in France or abroad, or from public or private research centers.
L'archive ouverte pluridisciplinaire HAL, est destinée au dépôt et à la diffusion de documents scientifiques de niveau recherche, publiés ou non, émanant des établissements d'enseignement et de recherche français ou étrangers, des laboratoires publics ou privés. 


\title{
Enhanced Spectrally Aware RF front end Receiver under Non-linearity
}

\author{
Nicolas Grollier ${ }^{1}$, Sébastien Houcke ${ }^{1}$, Michaël Pelissier ${ }^{2}$ \\ ${ }^{1}$ Institut Mines-Telecom, IMT Atlantique, Lab-STICC, UMR CNRS 6285, UBL, F-29238 Brest, France \\ Email: \{nicolas.grollier,sebastien.houcke\}@imt-atlantique.fr \\ ${ }^{2}$ CEA - LETI, University Grenoble Alpes, F-38000 Grenoble, France \\ Email: michael.pelissier@cea.fr
}

\begin{abstract}
In RF receivers nonlinearities are inherent to analog processing. This is the result of strong signals presence close to the bandwidth of the signal of interest, or a power excess of this signal. Both cases greatly deteriorate the receiver bit-error-rate. Methods to compensate for nonlinearities consist in equalizing resulting intermodulation terms by predistortion or a subtraction mechanism. In this article, we propose a proof of concept for a novel technique to limit the harmful effects of nonlinearities. It consists in making a measure of intermodulation terms power, thanks to a cyclostationary sensing mechanism. And then, to dynamically adapt receivers parameters to make it works in a linear regime. We detail principles of that method and develop the theoretical analysis of detection. Thanks to simulations, we show that this method is reliable and allows a nonlinearity detection 16dB below the compression point. This work was conducted in the scope of high end radio such as professional mobile radios (PMR) receiver.
\end{abstract}

\section{INTRODUCTION}

Current technologies of private communication systems PMR offer far less download speed than commercial technologies such as $4 \mathrm{G}$. Hence, the FITNESS project (see [1]) aims to take into account new users requirements and prepare the future of PMR in Europe and in the world. It paves the way towards high performance PMR based on Long-Term Evolution (LTE). New functionalities requested by mission critical markets are added, while preserving backwards compatibility with existing narrowband PMR systems. In this work, we focus on the narrowband receiver with stringent specifications, and more particularly on a method to make it compliant with the four European PMR norms in terms of receiver selectivity.

The main issue of PMR receivers is to deal with a dynamic up to $141 \mathrm{~dB}$ with channel bandwidth of $12.5 \mathrm{kHz}$. This specification is particularly severe, as we consider a fully integrated receiver (RX). Then, tight linearity and gain requirements lies on the front-end. To manage it, a new low-IF reconfigurable architecture has been defined. However, in presence of unwanted strong signals close to the signal of interest (SOI), some requirements may not be hold and the SOI may be affected. This degradation is the result of nonlinearities caused by RF components such as Low Noise Amplifier (LNA) or mixers (see [2]) used outside of their linear region. It might manifest through saturation, and presence of intermodulation products and harmonics. Indeed, as developed in [3], in presence of an additive unwanted strong signal with the SOI, some intermodulation distortion terms can be created and lay in the bandwidth of the SOI. In the following development, unwanted signals are referred as blockers, jammers or interferers.

The idea to compensate for nonlinearities is tackled by many ways. [4] proposes a training approach thanks to pilot signals. But, it requires to process baseband signals and interpret the received data flow, which is time consuming. Most advanced ways to compensate for third order intermodulation distortion (IMD3) products are developed in [5], [6]. Those methods rely on clever mechanisms to model the IMD3 products and then subtract it from the received signal. In [5] Valkama et al. propose a digital approach which requires very selective narrowband filter. ADCs sampling frequency also has to be high enough to respect Shannon's condition for interferers signals. This last requirement is very restrictive in our context since the considered bandwidth is fairly large. In a different manner, Keehr et al. use an analog cubic term generator to equalize the IMD3 products in [6]. However, as the RX has to be compliant with existing PMR standards, our RX architecture can't be modified. This solution also requires additional analog components and thus consumes more power than the original RX architecture. Those papers show that it is possible to almost completely remove the IMD3 terms at the cost of complex digital operations. Another strategy was chosen in [7]. The principle is to estimate the IMD3 terms thanks to a sensing mechanism, and subtracts it from samples of the SOI. So, IMD3 terms just have to be measured, and not longer to be modeled as in other approaches. But, each of these studies left aside the fact that powerful IMD3 harmonics are created only when interferers are strong, and close to the maximum dynamic.

In this paper, we propose a novel method to decrease IMD3 products power, based on the idea that the RX linearity could be improved thanks to dynamic range real-time tuning. In addition to pure analog design, a sensing mechanism could 


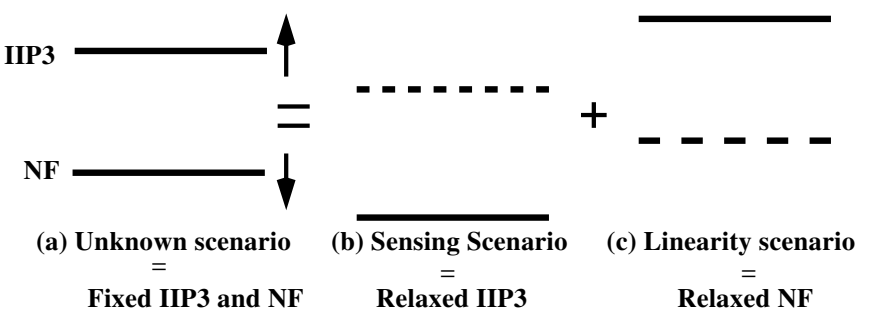

Fig. 1. Sensing scenario cases

be performed by digital processing means. That way, the RX could be partially aware of its spectral environment and could dynamically adapt its parameters thanks to a quick feedback loop. The detection is based on cyclostationary properties of IMD3 harmonics, which only appear when the RX works close to its nonlinear regime. Principles of this new method are developed in section II. The nonlinear system model, as well as principles of cyclostationarity, are presented in section III. In section IV, the cyclostationary analysis is derived and binary hypothesis test is defined. A numerical analysis and simulations are proposed in the section $\mathrm{V}$, and section VI concludes the paper.

\section{Digital Adaptive Automatic Gain Control}

In fig.1a, the unknown scenario corresponds to a classic receiver. In such a case, the RX parameters are fixed. The input interception point of third order (IIP3) is a quantity which serves as a comparison of linearity of different circuits. It has to be the highest as possible, in order to make the receiver as linear as possible. The noise figure (NF) classically describes the noise added by the analog RX to the received signal. The lower the NF, the better the signal-to-noise-ratio (SNR). However, NF and IIP3 are linked by the RX gain, and the maximization of IIP3 could be done only at the trade-off of a higher NF (see Friis formula and its nonlinear counterpart for cascaded IIP3 in [2]). We clearly understand the possible improvement if those parameters could be changed separately, which is exactly what we propose to do. Indeed, in the sensing scenario (fig.1b), we consider that the SOI is the only signal in presence (i.e. no jammers) and has a low power. In that particular case, the NF and IIP3 could be decreased to improve the SNR. Indeed, no jammer is present, the IIP3 requirement could be relaxed. In the linearity scenario (fig.1c), two cases could happened:

- the SOI is the only signal in presence (i.e. no jammers) and has a strong power,

- the SOI and jammers are present.

In both cases, we are not limited by sensitivity but rather by the RX linearity. So, the RX gain could be decreased to increase the IIP3, to allow a better linearity at the cost of a NF degradation. Moreover, it would be of some use to know if the RX is close to work in its nonlinear region, and identify the reason.

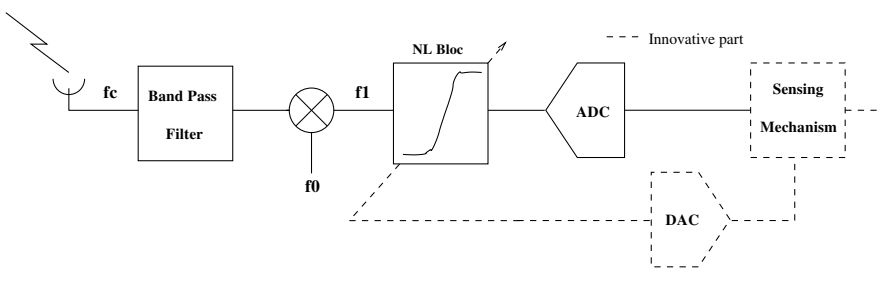

Fig. 2. PMR Receiver scheme

If the receiver is able to detect in which scenario it has to work, it could improve significantly its dynamic by applying a quick feedback loop to set up its parameters. This principle is depicted in fig.2, which represents a classic RX with the innovative part in dashed lines. To develop such a RX, the first step is to be able to detect in which scenario the RX is. This is the goal of the paper. So, the sensing mechanism allows us to: a) detect the working region of the RX (i.e. linear or nonlinear mode); b) detect the presence of interferer; c) identify the reason of the nonlinear behavior : if its is due to the interferer or due to a powerful SOI.

Let us now focus on the sensing mechanism, which has to be performed with very few information on the received signal. We also assume that neither the signal power nor the noise power are known. Blocker's potential carrier frequency and its modulation types are assumed to be known, and described in standards. Obviously, the algorithm sensitivity has to be high enough to detect the nonlinear behavior signatures. Several techniques of spectrum sensing, that are used in cognitive radios, are summarized in [8]. However, simples detectors (energy detection, waveform based-sensing, match filter) requires additional knowledge than our hypothesis. In particular, the noise power has to be known to use the energy detector. Moreover, this approach is not adapted to detect a specific interferer. In a same way, the transmission rate or at least the emission filter are required to use the match filter method. That is why we consider a detector based on received signal cyclostationary property in our context.

Based on the detection criterion, developed in [9], we propose to track several specific parameters which shows saturation or jammer presence. In this article, we propose the sensing algorithm and we demonstrate by simulation the efficiency of this concept.

\section{SYSTEM MODEL}

Our goal is now to be able to identify in which regime the RX works. We propose a novel sensing algorithm based on communication signal cyclostationary properties. We suppose a perfect synchronization between emitter and receiver in phase and frequency. That way, the RX signal frequencies are perfectly known and there is no phase noise. We also suppose that there is no I/Q imbalance, since this problem has several solutions proposed in the state-of-the-art. In the following, we consider the case of a nonlinear RX front-end of narrowband PMR signals. We only consider a model of third 
order, since the even-order harmonics lay far from SOI carrier frequency and thus can be discarded by a filtering operation. This simplification holds if we consider that higher odd-order distortion are far less powerful than third-order distortion. The selection of the SOI and amplification are performed before a digitization, which is realized at an intermediate frequency $f_{1}$ (see fig.2).

\section{A. Received signal model}

Let us define the base-band model of communication signals, where $T$ is the symbol period, $h(t)$ is a waveform lowpass filter and $s_{i, k}$ are the random complex symbols:

$$
z_{i}(t)=z_{i R}(t)+j z_{i I}(t)=\sum_{k=1}^{N} s_{i, k} h(t-k T)
$$

$z_{i R}(t)$ and $z_{i I}(t)$ are the real and imaginary parts of the baseband signal $z_{i}(t)$. The index ' $i$ ' take the values in $\{1,2\}$ and stands for useful and jammer signal respectively. Signal $x_{i}(t)$ at carrier frequency $f_{i}$ can be expressed as:

$$
y_{i}(t)=\Re\left[z_{i}(t) e^{j 2 \pi f_{i} t}\right]
$$

The SOI is at intermediate frequency such as $f_{1}=f_{c}-f_{0}$, where $f_{c}$ is the channel frequency before down conversion, as shown in fig. 2 .

We assume the following expression of the received signal, which is a combination of the useful signal and another PMR signal in an adjacent channel :

$$
x(t)=\sum_{i=1}^{2} y_{i}\left(t-\Delta_{t}\right)
$$

In the following parts, we take the propagation delay $\Delta_{t}$ in eq.(3), equal zero. A short development shows that this hypothesis has no impact on the method, and does not lead to a loss of generality.

\section{B. Nonlinear Model}

The nonlinear RX, which input is $x(t)$ defined in eq.(3), is classically expressed as a polynomial (see [3], [7]):

$$
p(t)=\alpha_{1} x(t)+\alpha_{2} x(t)^{2}+\alpha_{3} x(t)^{3}+n(t)
$$

Where $\alpha_{k}, k \in\{1,2,3\}$ are characteristics of the RX front-end and $n(t)$ is an additive white Gaussian noise (AWGN).

In this paper, the $\alpha_{2}$ coefficient is assumed to be 0 , since harmonics created by even coefficients are located at twice the frequency of input signal. Thus, there is no harmonic leaks at $f_{1}$. Such a harmonics are suppressed by a filter before demodulation. As in [7], $\alpha_{3}$ is obtained thanks to the relation eq.(5):

$$
\alpha_{3}=-\frac{4}{3} \frac{\alpha_{1}}{I I P 3^{2}}
$$

where IIP3 is the RX Input Interception Point of third order. Coefficients of $p(t)$ are extracted from practical measurements on the PMR receiver demonstrator conceived in CEA labs. The input-output representation of $p(t)$ with those parameters is

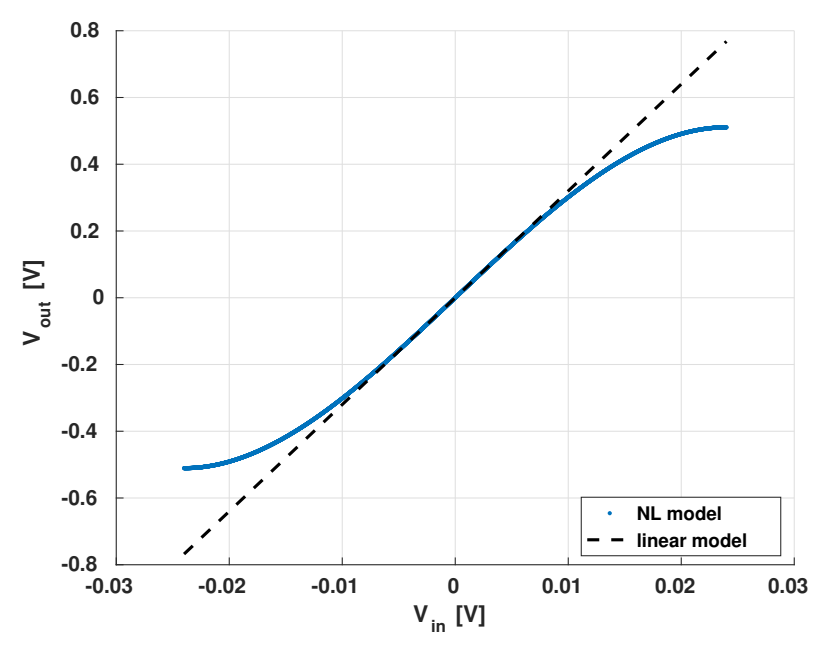

Fig. 3. $V_{\text {in }} / V_{\text {out }}$ representation of a real PMR receiver front-end non linear model

displayed in fig.3. In this article, the whole RX is modeled as a nonlinear system. This assumption holds, thanks to the equivalent of the Friis formula (see [2]) for nonlinear counterpart.

The substitution of eq.(3) into eq.(4) leads to the complete formula of harmonic creation for the nonlinear model. All the terms are listed in [3], but $\alpha_{2}$ is not considered here. We focus on the baseband representation of the received signal in a given subband of interest. The SOI in this subband is given by:

$$
\begin{aligned}
p(t)= & \operatorname{Re}\left\{\left(\alpha_{1} z_{1}(t)+\frac{3 \alpha_{3}}{2} z_{1}(t)\left|z_{1}(t)^{2}\right|\right.\right. \\
& \left.\left.+3 \alpha_{3} z_{1}(t)\left|z_{2}(t)\right|^{2}\right) e^{j 2 \pi f_{1} t}\right\}
\end{aligned}
$$

In the linearity scenario context, we consider that the SOI is much smaller than the blocker, so the term in $z_{1}(t)\left|z_{1}(t)^{2}\right|$ can be neglected. The term proportional to $\left|z_{2}(t)^{2}\right|$ becomes dominating as blocker's power comes high, and may affect the SOI. Hence, in the linearity scenario, coefficients $\alpha_{k}, k \in$ $\{1,3\}$ have to be modified to overcome the blocker's influence.

\section{Cyclostationarity Bases}

In this part, we derive the cyclostationary bases and principles of the sensing algorithm. A complete explanation on cyclostationary concept can be found in [10]. The main idea is that almost every communication signal is cyclostationary. That way, the autocorrelation function defined in eq.(7) is periodic in time.

$$
R_{z_{2}}(t, \tau)=E\left[z_{2}(t) z_{2}^{*}(t-\tau)\right]
$$

Here, $E[$.$] is the expectation operator and (.)* is the complex$ conjugate operation. As a periodic function, $R_{z_{2}}(t, \tau)$ admits a decomposition in Fourier series, which is given by:

$$
R_{z_{2}}(t, \tau)=\sum_{\alpha \in I_{z_{2}}} R_{z_{2}}^{\alpha}(\tau) e^{j 2 \pi \alpha t}
$$


Let $I_{z_{2}}=\left\{\frac{k}{T}, k \in \mathbb{Z}\right\}$ be the set of $z_{2}(t)$ cyclic frequencies. Fourier coefficients $R_{z_{2}}^{\alpha}(\tau)$ are called cyclic autocorrelation functions (CAF), where $\alpha$ is a specific cyclic frequency. The CAF theoretical expression is the Fourier transform of $R_{z_{2}}(t, \tau)$.

$$
R_{z_{2}}^{\alpha}(\tau)=\lim _{T_{\alpha} \rightarrow \infty} \frac{1}{T_{\alpha}} \int_{0}^{T_{\alpha}} R_{z_{2}}(t, \tau) e^{-j 2 \pi \alpha t} d t
$$

With that representation, $z_{2}(t)$ is cyclostationary and its cyclic frequencies are multiple of $1 / T$. In eq.(8), for $k>1$ the CAF of $z_{2}(t)$ are 0 since their magnitude drops quickly.

The classic method to check if $\alpha$ is a cyclic frequency consists in estimating the corresponding CAF of the received signal, and measure its power. The natural estimator of CAF is defined in eq.(10) below:

$$
\hat{R}_{z_{2}, N}^{\alpha}(l)=<z_{2}(k) z_{2}^{*}(k-l) e^{-j 2 \pi \alpha k}>
$$

In eq.(10), the expectation is replaced by a temporal average operator defined as $\langle\rangle=.\frac{1}{N+1} \sum_{k=-N / 2}^{N / 2}($.$) . The notation$ $\hat{R}_{z_{2}, N}^{\alpha}(l)$ stands for the estimate of $R_{z_{2}}^{\alpha}\left(l T_{s}\right)$, with $T_{s}$ the sampling period.

However, PMR signals which uses among others the QPSK or 4QAM modulations, can't be detected by order two estimators such as eq.(10). Hence, we propose to use the detection criterion developed in [9] and bring out in eq.(11):

$$
\hat{J}_{L, N}(\alpha)=\frac{\hat{C}_{N}^{\alpha}(L)}{\hat{C}_{N}^{\beta}(L)}
$$

where:

$$
\hat{C}_{N}^{\eta}(L)=\frac{1}{L+1} \sum_{l=0}^{L}\left|\hat{R}_{z_{2}, N}^{\eta}(l)\right|^{2}
$$

The criterion $\hat{J}_{L, N}\left(2 f_{2}\right)$ allows us to detect a signal at $2 f_{2}$, even QPSK modulated. Indeed, for that particular case, $\hat{C}_{N}^{2 f_{2}}(L)$ and $\hat{C}_{N}^{\beta}(L)$ do not converge to zero in the same way. Neither $2 f_{2}$ is a cyclic frequency, nor $\beta$, but this particular behavior allows the detection.

Another particularity of this criterion is that it doesn't need a long observation time to perform the detection. This is not a kind of energy level measure, but rather a measure of a convergence behavior difference. It appears that even with a very short observation duration, this behavior remains similar, making $\hat{J}_{L, N}\left(2 f_{2}\right)$ almost independent of $\mathbf{N}$. This behavior is shown for a set of representative values of $\mathrm{N}$ in table I.

A theoretical detection threshold $\Gamma$ could be estimated from eq.(11). Let us consider that $z_{2}(t)$ is an AWGN signal to

TABLE I

Detection Probability $\left(P_{D}\left(2 f_{2}\right)\right)$ IN FUnCtion of N, FOR A FALSE ALARM PROBABILITY $P_{f a}=5 \%$, AND SNR $=2 \mathrm{~dB}$

\begin{tabular}{|c|c|c|c|c|}
\hline Number of symbols $(\mathrm{N})$ & 6 & 32 & 128 & 512 \\
\hline$P_{D}\left(2 f_{2}\right)[\%]$ & 0.827 & 0.833 & 0.843 & 0.832 \\
\hline
\end{tabular}

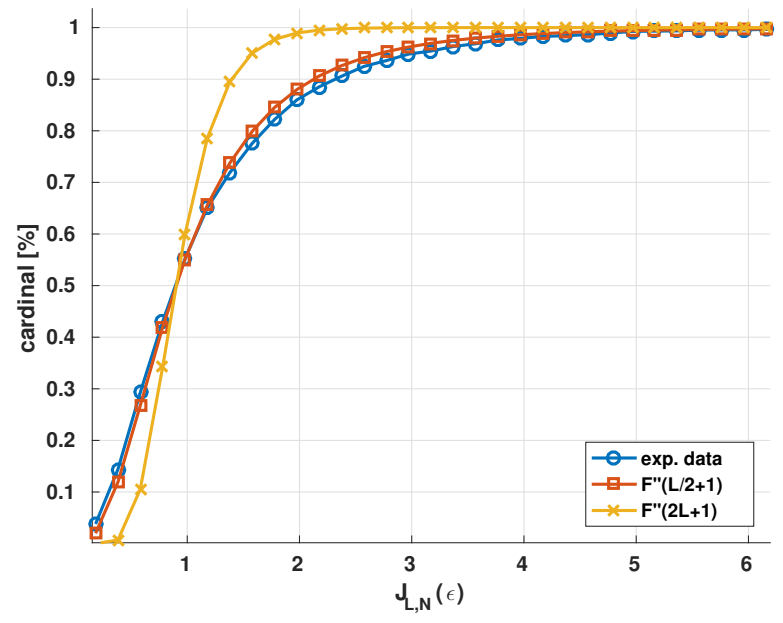

Fig. 4. Cumulative distribution function comparison for experimental data under $H_{0}$ and doubly non central Fisher laws of $2 L+1$ or $\lfloor L / 2\rfloor+1$ degrees of freedom, where $L=20$

determine the $P\left[\hat{J}_{L, N}\left(2 f_{2}\right) \Gamma \geq \mid H_{0}\right]$. Under this hypothesis, both $\hat{C}_{N}^{2 f_{2}}(L)$ and $\hat{C}_{N}^{\beta}(L)$ are a sum of modulus complex normal values at the power two. Therefore, nominator and denominator of $\hat{J}_{L, N}\left(2 f_{2}\right)$ follows unnormalized $\chi^{2}$ distributions of $2 L+1$. For that reason the criterion follows a doubly non central Fisher distribution of $2 L+1$ freedom degrees.

\section{Nonlinear Detection BASEd on a CyClOSTATIONARY ANALYSIS}

In this section, we develop the cyclostationary analysis of the signal $p(t)$ after digitization.

\section{A. Cyclostationary analysis}

Considering the linearity scenario, we assume that a powerful interferer $y_{2}(t)$ is present. We use the criterion eq.(11) to detect its presence and track its CAF power at $2 f_{2}$. Now, we need to understand the impact of nonlinearities on cyclostationarity properties of the received signal. To determine if the RX works in a nonlinear mode, we propose to realize a short cyclostationary analysis.

The eq.(4) is a sum of harmonics of frequency $f_{1}, f_{2}$, $2 f_{1}-f_{2}, 2 f_{2}-f_{1}, 3 f_{1}$ and $3 f_{2}$ (see [3]). Hence, a theoretical search of cyclic frequencies (using eq.(9) and eq.(4)) leads to a 81 terms expression, which are not detailed here for brevity consideration. However, we noticed the presence of powerful CAFs at cyclic frequencies $4 f_{1}$ and $4 f_{2}$. Their expression is developed in eq.(13):

$$
\hat{R}_{p}^{4 f_{i}}(l)=<\alpha_{1} \alpha_{3}\left|y_{i}(k)\right|^{2}\left|y_{i}(k-l)\right|^{2} e^{-j 2 \pi 4 f_{i} k}>
$$

with $i \in\{1,2\}$. The expression $\hat{R}_{p}^{4 f_{i}}(l)$ is homogeneous to a high order moment due to the nonlinear transformation. Moreover, as a self product, it produces one of the greatest energy.

So, we propose to monitor the criterion set $\mathcal{M}=$ $\left\{\hat{J}_{L, N}\left(2 f_{2}\right), \hat{J}_{L, N}\left(4 f_{1}\right), \hat{J}_{L, N}\left(4 f_{2}\right)\right\}$. That information is 


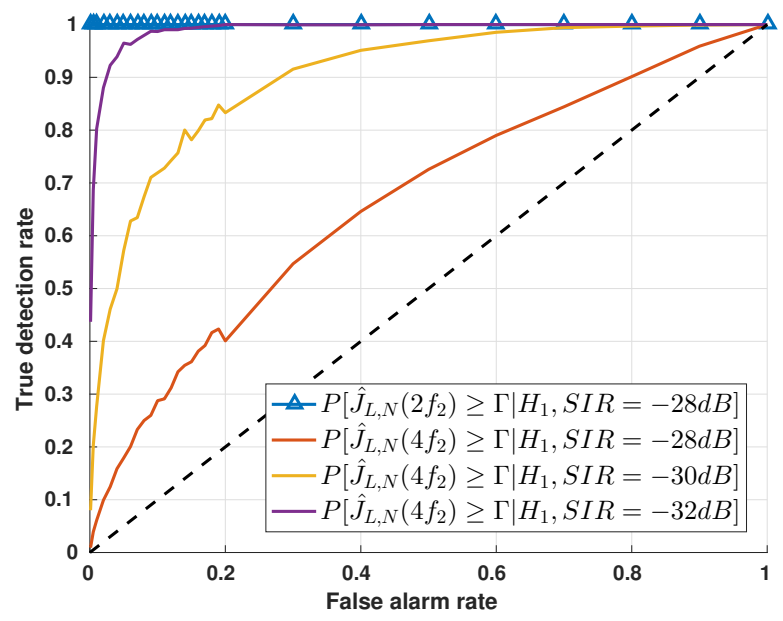

Fig. 5. ROC curves at fixed $\mathrm{SNR}=2 \mathrm{~dB}, \mathrm{SIR}=30 \mathrm{~dB}$, power of test for monitored CAFs of set $\mathcal{M}$

enough to select the corresponding (b) or (c) scenario described in fig.1.

\section{B. Binary Hypothesis Testing}

Each element of $\mathcal{M}$ is tested accordingly to a NeymanPearson approach [11]. A constant false alarm threshold $\Gamma$, is set to satisfy $P_{f a}=P\left[\mathcal{M} \geq \Gamma \mid H_{0}\right]$. The test is the following one:

$$
H:\left\{\begin{array}{l}
H_{0} \rightarrow p(t)=\operatorname{Re}\left\{\alpha_{1} z_{1}(t)+\alpha_{3} z_{1}^{3}(t)\right\}+n(t) \\
H_{1} \rightarrow p(t)=\operatorname{Re}\left\{\alpha_{1} x(t)+\alpha_{3} x(t)^{3}\right\}+n(t)
\end{array}\right.
$$

Here, $H_{0}$ denotes the absence of blocker and $H_{1}$ denotes its presence. However, in eq.(14) the $H_{0}$ hypothesis corresponds to SOI presence plus noise, instead of noise only as assumed in the previous section.

Hence, we showed trough simulations that $P\left[\hat{J}_{L, N}\left(4 f_{2}\right) \geq\right.$ $\left.\Gamma \mid H_{0}\right]$ don't follow a doubly non central Fisher law of $2(L+1)$ freedom degrees. Indeed, as we can see in fig.4, the cumulative distribution function of experimental data seems to follow a $\lfloor L / 2\rfloor+1$ doubly non central Fisher law. The most likely explanation would be that real and complex parts of the CAFs $\hat{R}_{p, N}^{\varepsilon}(l)$ are not independent. The theoretical justification of such a dimension reduction is under demonstration, and will be presented in a future work.

\section{Simulation And Numerical Analysis}

In this section, we develop our simulation results, and we show that we are able to identify precisely in which scenario the RX is. We also show thanks to simulations, that the proposed detection method answers the problematics of rapidity, sensibility and reliability.

\section{A. Experimental Conditions}

To make sure that there is no cyclic frequency overlap, we set a sampling rate $f_{s}=10 f_{c}$. The shaping filter $h(t)$ is

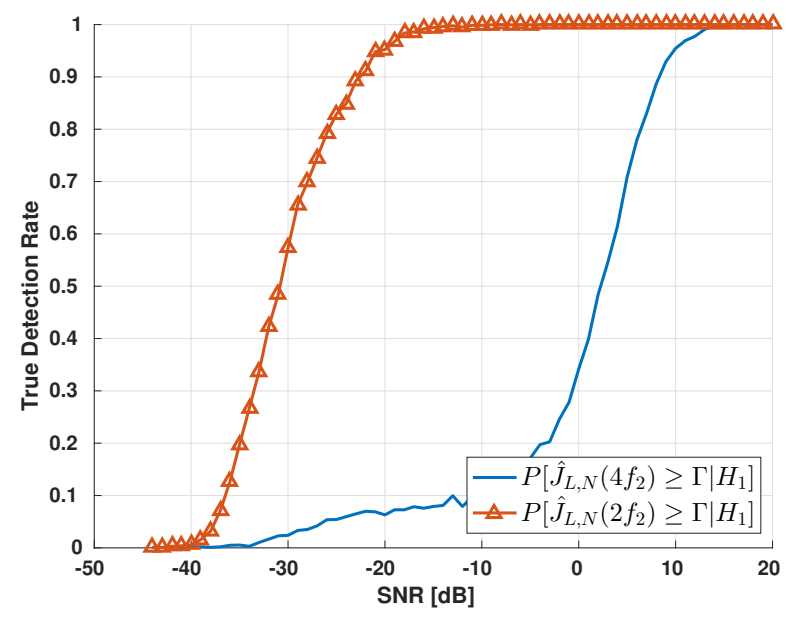

Fig. 6. criterion probability of true detection in function of SNR level, with a fixed threshold $P_{f a}=5 \%$ and SIR $=-30 \mathrm{~dB}$

defined as a square-root raised cosine of period $T=2.5 T_{1}$, a roll-off at 0.8 and span at 6 symbols. As detailed in section III, nonlinear model coefficients are set thanks to measurements on an experimental RX. The RX gain is fixed at $32 d B$, when the input interception point of order $3 I I P 3=3.6 \mathrm{dBm}$. Blocker and useful signals are in adjacent channel, and the blocker carrier frequency is defined as $T_{2}=T_{1} / 2.4$. Both SOI and blocker are 4QAM symbols, independent and identically distributed. $N$ the number of symbols is set at 32, which is few, as discussed in the previous section. We set L, the number of delays used in the $\hat{J}_{L, N}(\alpha)$ estimation at 25, in order to limit the calculation cost. We defined the SOI power $\sigma_{y_{1}}^{2}$ at $-106.0 \mathrm{dBm}$. 2048 Monte Carlo runs are performed to estimate our method's performance.

\section{B. Results}

We begin this section with the power of the test analysis, for each element of $\mathcal{M}$. Tests at frequencies $4 f_{1}, 4 f_{2}$ have same power. So, results are provided only for the tests at frequencies $2 f_{2}$ and $4 f_{2}$. To answer the nonlinear sensibility issue, we designed a simulation which corresponds to the linearity scenario (i.e. scenario 1c) to produce nonlinear harmonics of different power. The signal-to-noise-ratio (SNR) is set at $2 \mathrm{~dB}$ and the signal-to-interference-ratio (SIR) is defined as summed up in table II.

TABLE II

SIR VALUES AND CORRESPONDING DETECTION PROBABILITY, FOR A $P_{f a}=5 \%$

\begin{tabular}{|c|c|c|}
\hline SIR [dB] & Blocker Range $\left[\mathrm{mV}_{\mathrm{pp}}\right]$ & $P_{D}\left(4 f_{2}\right)[\%]$ \\
\hline-28 & 4.0 & 23 \\
\hline-30 & 4.5 & 65 \\
\hline-32 & 5.0 & 95 \\
\hline
\end{tabular}


In fig.5, we draw the power of the test using criterion $\hat{J}_{L, N}\left(2 f_{2}\right)$ and $\hat{J}_{L, N}\left(4 f_{2}\right)$. Those curves represent the receiver operating characteristic (ROC) for the two criteria. For each blocker power, we draw the criterion $\hat{J}_{L, N}\left(2 f_{2}\right)$, which allows us to reach $100 \%$ of good detection. The $P\left[\hat{J}_{L, N}\left(2 f_{2}\right) \geq \Gamma \mid H_{1}\right]$ ROC for a SIR set at $-28 \mathrm{~dB}$, is the only one represented for convenience. Hence, with this simulation parameters the blocker presence could be determined with high confidence. We can see in table II, that a good detection is obtained for the higher SIR, and that the detection rate decreases with the SIR. It is clear that as the $\mathrm{RX}$ is weakly nonlinear and the $4 f_{2}$ harmonic power is small.

To answer to the nonlinear harmonic detection reliability against noise, we defined a $P_{f a}=5 \%$. The SIR is defined at $-30 \mathrm{~dB}$, to remain slightly nonlinear. We measure the detection probability for several values of SNR. This analysis is illustrated through fig.6. As we can see, blocker detection gives a perfect detection if the SNR is greater than $-15 \mathrm{~dB}$. Which means that, the blocker is perfectly detected $30 \mathrm{~dB}$ before the nonlinear harmonic. As the RX works in weakly nonlinear mode, the $4 f_{2}$ harmonic is small in comparison to the noise floor. Hence, if the SNR decreases, the detection probability increases to reach $95 \%$ of good detection for a SNR of $10 \mathrm{~dB}$.

Fig.7 is dedicated to illustrate the sensibility of our detector. This detection curve was obtained for a SNR of $2 \mathrm{~dB}$ and a $P_{f a}=5 \%$. The true detection rate grows quickly when the SIR comes higher than $27 \mathrm{~dB}$, which is consistent with remarks on fig.6. As $P_{I I P 3}=3.6 \mathrm{dBm}$, the corresponding compression point is $P_{C 1 d B}=-6 \mathrm{dBm}$. A perfect detection is obtained for a SIR set at $-32 \mathrm{~dB}$, which corresponds to a $P_{D}\left(4 f_{2}\right)=-22 \mathrm{dBm}$. Hence, the detection is performed $16 \mathrm{~dB}$ before the $P_{C 1 d B}$. Our detector allows a detection before the system comes in a strong nonlinear region. So, our method could be used to adjust accordingly the dynamic range of the RX, before the degradation of its performance.

\section{CONCLUSION}

In this article, we developed a proof of concept of a spectrally aware PMR receiver in order to enhance its linearity. We point out pros and cons of state-of-the-art methods, and then develop the concept of our receiver. We goes on with the nonlinear system model and order two cyclostationary detector principles. It allows us to develop a binary hypothesis test and to derive the theoretical background in a non linear context. Finally, we showed through simulations that this concept fulfills the RX requirements. The detection metrics proves the efficiency and reliability of the nonlinear components detection. As the detection method is very fast and sensitive, we consider that a further analysis of this new concept is interesting. In a future work, we propose to complete this study with the design and analysis of the feedback loop.

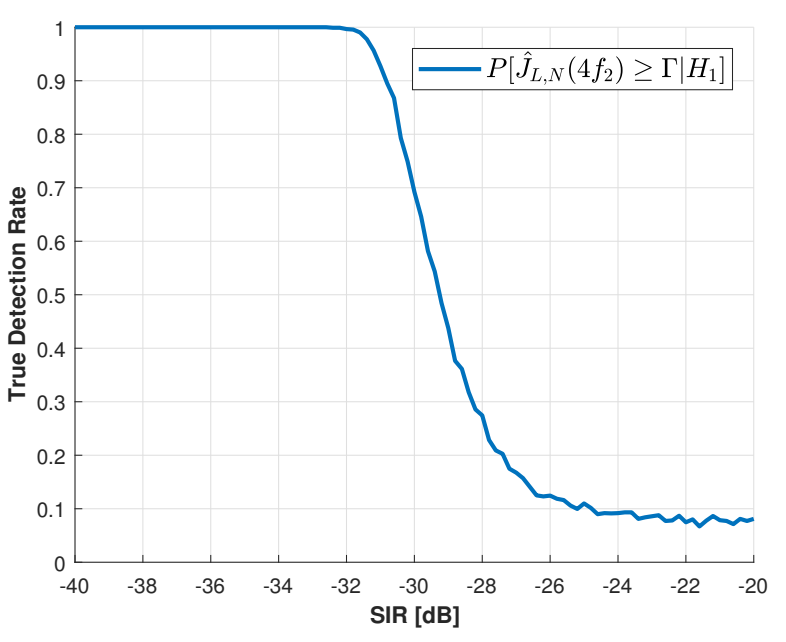

Fig. 7. criterion probability of true detection in function of SIR level, with a fixed threshold $P_{f a}=5 \%$ and SNR $=2 \mathrm{~dB}$

\section{ACKNOWLEDGMENT}

This work was realized in cooperation with CEA, and funded in the scope of the FITNESS project (see [1]). The authors would like to thank the FITNESS partners: Airbus Defense, MKR-IC, BeSpoon, CEA, IMS, NXP, ISEP and ADS, for their cooperation.

\section{REFERENCES}

[1] "Fitness scope," http://lisite.isep.fr/minarc/minarc-projects/fitness/, accessed: 2017-02-28

[2] B. Razavi, RF Microelectronics (2Nd Edition) (Prentice Hall Communications Engineering and Emerging Technologies Series), 2nd ed. Upper Saddle River, NJ, USA: Prentice Hall Press, 2011.

[3] Q. Zou, M. Mikhemar, and A. H. Sayed, "Digital compensation of cross-modulation distortion in software-defined radios," IEEE Journal of Selected Topics in Signal Processing, vol. 3, no. 3, pp. 348-361, June 2009.

[4] R. Myer, "Automatic reduction of intermodulation products in high power linear amplifiers," Apr. 1 1986, uS Patent 4,580,105. [Online]. Available: https://www.google.com/patents/US4580105

[5] M. Valkama, A. S. hagh ghadam, L. Anttila, and M. Renfors, "Advanced digital signal processing techniques for compensation of nonlinear distortion in wideband multicarrier radio receivers," IEEE Transactions on Microwave Theory and Techniques, vol. 54, no. 6, pp. 2356-2366, June 2006.

[6] E. A. Keehr and A. Hajimiri, "Equalization of third-order intermodulation products in wideband direct conversion receivers," IEEE Journal of Solid-State Circuits, vol. 43, no. 12, pp. 2853-2867, Dec 2008.

[7] E. Rebeiz, A. S. H. Ghadam, M. Valkama, and D. Cabric, "Spectrum sensing under rf non-linearities: Performance analysis and dsp-enhanced receivers," IEEE Transactions on Signal Processing, vol. 63, no. 8, pp. 1950-1964, April 2015.

[8] T. Yucek and H. Arslan, "A survey of spectrum sensing algorithms for cognitive radio applications," Commun. Surveys Tuts., vol. 11, no. 1, pp. 116-130, Jan. 2009. [Online]. Available: http://dx.doi.org/10.1109/ SURV.2009.090109

[9] N. Grollier and S. Houcke, "On carrier qpsk signal detector based on second order cyclic-moments," in SUBMITTED to 2018 IEEE Wireless Communications and Networking Conference (WCNC), April 2018.

[10] W. A. Gardner, Statistical spectral analysis, A non probabilistic theory. Englewood Cliffs,New Jersey: Prentice-Hall, 1988.

[11] S. M. Kay, Fundamentals of Statistical Signal Processing: Practical Algorithm Development. Pearson Education, 2013, vol. 3. 